\title{
Influencing the binding configuration of sucrose in the active sites of chicory fructan 1-exohydrolase and sugar beet fructan 6-exohydrolase
}

\author{
Katrien Le Roy ${ }^{1}$, Willem Lammens ${ }^{1,2}$, André Van Laere ${ }^{1}$ and Wim Van den Ende ${ }^{1}$ \\ ${ }^{1}$ K. U. Leuven, Laboratory of Molecular Plant Physiology, Kasteelpark Arenberg 31, box 2434, B-3001 Leuven, Belgium; ${ }^{2}$ K. U. Leuven, Laboratory of \\ Biocrystallography, Herestraat O \& N II, box 822, B-3000 Leuven, Belgium
}

\begin{abstract}
Author for correspondence: Wim Van den Ende

Tel: ++32 16321952

Fax: ++3216321967

Email: wim.vandenende@bio.kuleuven.be
\end{abstract}

Received: 12 October 2007

Accepted: 7 January 2008

\section{Summary}

- The hydrolytic plant enzymes of family 32 of glycoside hydrolases (GH32), including acid cell wall type invertases (EC 3.2.1.26), fructan 1-exohydrolases (1-FEH; EC 3.2.1.153) and fructan 6-exohydrolases (6-FEH; EC 3.2.1.154), are very similar at the molecular and structural levels, but are clearly functionally different. The work presented here aims at understanding the evolution of enzyme specificity and functional diversity in this family by means of site-directed mutagenesis.

- It is demonstrated for the first time that invertase activity can be introduced in an S101 L mutant of chicory (Cichorium intybus) 1-FEH lla by influencing the orientation of Trp 82. At high sucrose and enzyme concentrations, a shift is proposed from a stable inhibitor configuration to an unstable substrate configuration.

- In the same way, invertase activity was introduced in Beta vulgaris 6-FEH by introducing an acidic amino acid in the vicinity of the acid-base catalyst (F233D mutant), creating a $\beta$-fructofuranosidase type of enzyme with dual activity against sucrose and levan.

- As single amino acid substitutions can influence the donor substrate specificity of FEHs, it is predicted that plant invertases and FEHs may have diversified by introduction of a very limited number of mutations in the common ancestor.

Key words: fructan, fructan exohydrolase (FEH), invertase, site-directed mutagenesis, sucrose.

New Phytologist (2008) 178: 572-580

(C) The Authors (2008). Journal compilation (C) New Phytologist (2008)

doi: 10.1111/j.1469-8137.2008.02386.x

\section{Introduction}

Polysaccharides are important biomolecules in plants, playing a role as reserve carbohydrate or as a structural component. The so-called glucans, polymers of glucose, are the most common and best studied plant polysaccharides; cellulose is the main component of the plant cell wall, while starch is the most important storage carbohydrate. However, not only glucans, also fructans, soluble sucrose-based polymers of fructose, are widespread alternative reserve carbohydrates. Fructans have been reported in various prokaryotes, fungi and algae, and accumulate in $15 \%$ of the flowering plants, including the economically important orders Poales and Asterales (Hendry \& Wallace, 1993).
In contrast to the uniform structure of microbial fructans, plant fructans show more structural diversity and are biosynthesized by different types of fructosyltransferases (Shiomi, 1989; Vijn \& Smeekens, 1999; Lasseur et al., 2006). Fructans are synthesized by an initial fructosyl transfer to one of the primary hydroxyl groups of sucrose, followed by further chain elongation (Van Laere \& Van den Ende, 2002). Different types of fructan molecules can be distinguished depending on the linkage type between the fructosyl residues (Lewis, 1993). Fructans in plants may be classified into two categories according to the position of the glucosyl residue. Fructans with a terminal glucosyl residue include the $\beta(2-1)$ type fructans (inulin, principally occurring in dicots), and the linear $\beta(2-6)$ (levan) or branched (graminan) $\beta(2-6)$ type fructans (as 
occurring in bacteria and monocots). Fructans with an internal glucosyl residue include the neo-inulin and neo-levan type (as occurring in Lolium perenne). In contrast to microbial fructans, the degradation of plant fructans appears to be catalysed exclusively by fructan exohydrolases (FEHs) releasing one terminal fructose molecule at a time using water as acceptor. Until now, no plant fructan endohydrolases have been reported.

Plant FEHs are more closely related to cell wall invertases than to vacuolar invertases (Van den Ende et al., 2000). Cell wall invertases are key metabolic enzymes involved in the regulation of sucrose partitioning, developmental processes such as pollen and seed development and the response to wounding and pathogen infection (Koch, 1996). Plant FEHs can be classified into 1-FEHs (inulinases, EC 3.2.1.153) and 6-FEHs (levanases, EC 3.2.1.154), according to the linkage type they hydrolyse. Some forms are able to degrade both $\beta(2-1)$ and $\beta(2-6)$ linkages and are termed 6\&1-FEHs (De Coninck et al., 2005; Kawakami et al., 2005). Rapid fructan breakdown by FEHs is widely recognized to be an essential process for sugar mobilization in fructan plants whenever carbon supply is needed by sink organs (Morvan-Bertrand et al., 2001; Asega and Carvalho, 2004). Different types of these FEHs have been fully characterized and cloned (Van den Ende et al., 2000, 2001, 2003a; Van Riet et al., 2006; Lothier et al., 2007). Interestingly, FEH activities were also detected in plants that do not accumulate fructans. Specific FEHs (lacking invertase activity) were cloned and/or purified from Beta vulgaris (6-FEH) and Arabidopsis thaliana (6-FEH and 6\&1-FEH) (Van den Ende et al., 2003b; De Coninck et al., 2005). The question why FEHs are present in plants that do not accumulate fructan substrates is still unanswered. It remains to be elucidated whether FEHs might be involved in signalling or other regulatory processes (De Coninck et al., 2005).

Unlike microbial FEHs that generally can hydrolyse sucrose as well (Vandamme \& Derycke, 1983), all plant FEHs purified so far seem to be unifunctional enzymes unable to degrade sucrose (De Roover et al., 1999; Verhaest et al., 2007). This observation might be related to the fact that for plants (in contrast to microorganisms) sucrose also represents the major carbon transport form between source and sink tissues and forms an important signal molecule and key role player in plant growth and development (Koch, 1996). Moreover, sucrose inhibits many FEHs in vitro, strongly suggesting that FEHs might be regulated by sucrose in vivo (De Roover et al., 1999; Van den Ende et al., 2003a; Lothier et al., 2007).

Plant invertases preferentially hydrolyse the $\alpha 1,2$ Glc-Fru linkage in sucrose, while plant FEHs have no invertase activity and only split terminal $\beta$ Fru-Fru linkages in fructans. The term ' $\beta$-fructofuranosidase' is used for enzymes that can hydrolyse both sucrose and fructans. Together with fructan biosynthetic enzymes, all these kinds of enzymes group together in the glycoside hydrolase family 32 (GH32) in the carbohydrate active enzymes database (Henrissat \& Davies, 1997; www.cazy.org). Family GH32 is combined with the related family GH68, harbouring bacterial invertases, levansucrases and inulosucrases, in the clan GH-J (Naumoff, 2001).

As both plant invertases and FEHs are believed to fulfil crucial functions during plant growth and development, our research focuses on fully explaining the difference in substrate specificity between these very related enzymes. This research is facilitated by elucidation of the three-dimensional structures of Cichorium intybus 1-FEH IIa (Verhaest et al., 2005, PDB code 1ST8) and $A$. thaliana cell wall invertase 1 (Verhaest et al., 2006, PDB code 2AC1). Both enzymes consist of an $\mathrm{N}$-terminal five-fold $\beta$-propeller domain and a $\mathrm{C}$-terminal domain formed by two $\beta$-sheets. A similar fivefold $\beta$-propeller domain is found in all other protein structures of the GH-J clan, as summarized by Verhaest et al. (2007). The active site is located in the $\beta$-propeller domain and is composed of three highly conserved acidic amino acids, one glutamate and two aspartate residues, acting as nucleophile, acid-base catalyst and transition-state stabilizer, respectively (Reddy \& Maley, 1990, 1996; Fig. 1).

The three-dimensional structure of chicory 1-FEH IIa in complex with its inhibitor sucrose was determined (Verhaest et al., 2007). These authors demonstrated that the binding of sucrose in the 'inhibitor configuration' in chicory 1-FEH IIa (in which the glucose moiety shows another orientation as the one observed in the levansucrase-sucrose complex) is determined by the specific orientation of a single amino acid, Trp82 of the highly conserved WSGSAT region. Furthermore, recent site-directed mutagenesis studies showed that a D239A mutant of a cell wall invertase of $A$. thaliana lost its capacity to degrade sucrose efficiently, while its intrinsic FEH activity appeared unaffected (Le Roy et al., 2007b), leading to the hypothesis that the FEHs occurring in $A$. thaliana might be considered as 'defective' invertases. To test this hypothesis further, we were challenged to (re)build invertase activity in FEHs, a process expected to be much more difficult than destroying invertase activity. The experiments were performed on two different types of FEH: chicory 1-FEH IIa strongly binding sucrose as an inhibitor; and B. vulgaris 6-FEH, which is not inhibited by sucrose.

\section{Materials and Methods}

\section{Cloning and site-directed mutagenesis}

Cichorium intybus L. 1-FEH IIa (Ci1-FEHIIa) and B. vulgaris 6-FEH (Bv6-FEH) were cloned into the pPICZ $\alpha A$ vector as described by Verhaest et al. (2004); Van den Ende et al. (2003b). Single amino acid substitutions were generated following the Quick Change protocol (Stratagene, La Jolla, CA, USA), using the pPICZ $\alpha A-C i 1-F E H I I a$ and pPICZ $\alpha A-B v 6-$ 
(a)

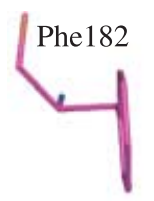

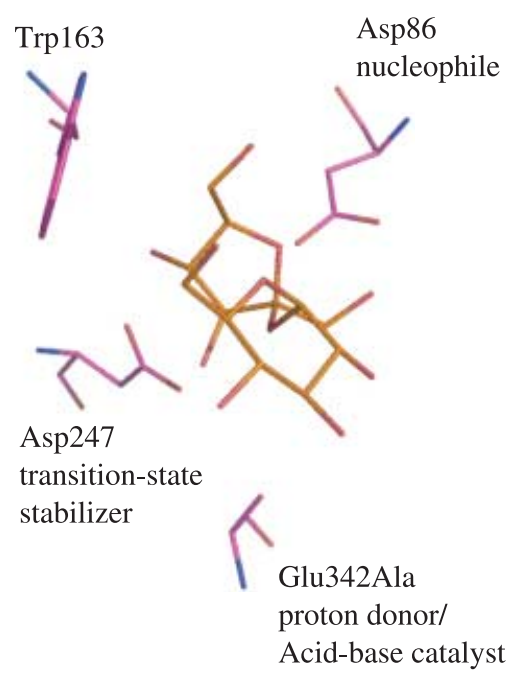

(b)

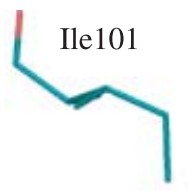

(c)

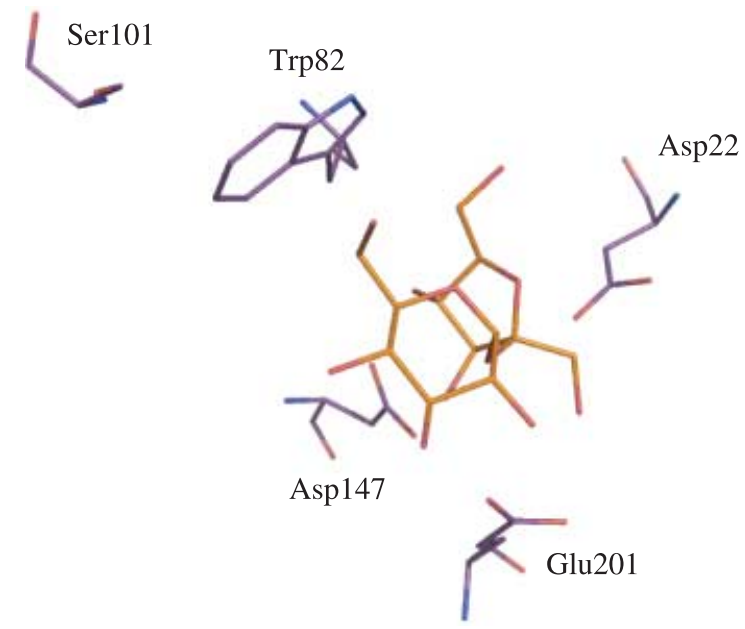

Fig. 1 Position of sucrose as a substrate or inhibitor in the active sites of (a) Bacillus subtilis levansucrase (PDB code 1PT2); (b) Arabidopsis thaliana cell wall invertase 1 (AtcwINV1); (c) Cichorium intybus 1-FEH Ila (PDB code 2ADD). The position of sucrose in AtcwINV1 is based on the known structural complex of $B$. subtilis levansucrase with sucrose. Figures were prepared with PyMOL (Delano, 2002).

FEH constructs as a template. For site-directed mutagenesis, the following forward oligonucleotide primers (and complementary reverse primers) were used: 5 '-CCTTAAATTAAGTTTGGACG ATACTCAATATGAG-3' (Bv6-FEH/F233D) and 5'-GTA CACCGGCCTCGATTCAAG-3' (Ci1-FEHIIa/S101L). The mutations are illustrated in bold. Further handlings were as described by Le Roy et al. (2007a).

\section{Heterologous expression and purification}

The methylotrophic yeast Pichia pastoris was used for extracellular gene expression as described by De Coninck $e t$ al. (2005). Purification of the recombinant Ci1-FEHIIa and Bv6-FEH wild-type and mutant proteins was as described by Le Roy et al. (2007a). Enzyme concentrations were measured using the Bradford method with bovine serum albumin as a standard (Sedmak \& Grossberg, 1977).

\section{Enzyme assays}

Appropriate aliquots of purified enzyme were mixed with different substrates (with a final concentration ranging from $1.0 \mathrm{~mm}$ to $1.0 \mathrm{M}$ ) in 50-mm sodium acetate buffer $\mathrm{pH} 5.0$ also containing $0.02 \%(\mathrm{w} / \mathrm{v})$ sodium azide. Chicory inulin (mean DP 30, Sigma-Aldrich, St Louis, MO, USA), 1-kestose (TCI Europe nv, Zwijndrecht, Belgium) and sucrose are commercially available substrates, bacterial levan (mean DP 72) and 6-kestose were generous gifts from Dr lizuka (lizuka et al., 1993). Reaction mixtures were incubated at $30^{\circ} \mathrm{C}$. For the reactions with sucrose, time points were $30 \mathrm{~min}, 1$ and $2 \mathrm{~h}$. For the other substrates, time points were 10, 20 and $30 \mathrm{~min}$, respectively. Total enzyme activity was determined by measuring the amount of released fructose by high-pressure anionexchange chromatography with pulsed amperometric detection (HPAEC-PAD, Dionex, Sunnyvale, CA, USA) (Van den Ende 
New

Phytologist

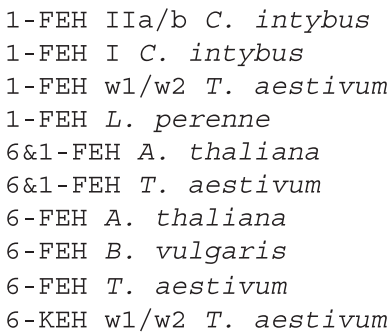

82

WSGSAT

LSGSAT

WTGSVT

WTGSTT

WSGSAT

WTGSAT

WSGSVT

WSGSIT

WSGSAT

WSGSAT

101
MLYTGS
ILYTGQ
IIYTGG
IIYTGG
ILYTGL
IIYTGL
ILYTGI
ILYTGI
LLYTGR
IIYTGV

Fig. 2 Multiple sequence alignment (using CLUSTALW) of the Trp82 and Ser101 regions in plant fructan exohydrolases and correlation with the inhibitory effect of sucrose. The following cDNA-deduced amino acid sequences (with GenBank accession numbers) were used for the alignment: chicory (Cichorium intybus) fructan 1-exohydrolase (1-FEH Ila) (AJ295033), 1-FEH Ilb (AJ295034) and 1-FEH I (AJ242538); Triticum aestivum 1-FEH w1 (AJ516025), 1-FEH w2 (AJ508387), 6\&1-FEH (AB089269), 6-FEH (AM075205), 6-KEH w1 (AB089271) and 6-KEH w2 (AB089270); Lolium perenne 1-FEHa (DQ016297); Arabidopsis thaliana 6\&1-FEH (AY060533) and 6-FEH (AB029310); Beta vulgaris 6-FEH (AJ508534).

\& Van Laere, 1996). Only data from the linear range were used and experiments were repeated three times with consistent results.

\section{Results and Discussion}

\section{Sucrose binding in FEHs: inhibitor vs substrate configuration}

The three-dimensional structure of chicory 1-FEH IIa in complex with its inhibitor sucrose revealed that sucrose binds in a different configuration compared with the substrate configuration, as observed in B. subtilis levansucrase (Meng \& Fütterer, 2003; PDB code 1OYG). The terminal fructosyl unit is positioned in a very similar way at the -1 subsite (Fig. 1), but the configuration of the glucosyl moiety of sucrose bound in chicory 1-FEH IIa at the +1 subsite is clearly different. Remarkably, Trp82 occupies a different orientation in 1-FEH IIa (Fig. 1c) compared with its structural homologues in B. subtilis levansucrase (Trp163; Fig. 1a) and AtcwINV1 (Trp82; Fig. 1b). Recently, site-directed mutagenesis studies clearly demonstrated that this specific orientation of Trp82 in chicory 1-FEH IIa is caused by the presence of the small, nonstacking Ser101 residue, allowing sucrose to bind in an alternative inhibitor configuration in the active site. Sucrose cannot be hydrolysed when it binds in the inhibitor configuration (Verhaest et al., 2007). Strikingly, most FEHs that are (very) weakly inhibited by sucrose are characterized by the presence of a hydrophobic residue (leucine, isoleucine, valine) at this Ser 101 position, stacking with the Trp 82 homologue and probably preventing sucrose from binding as an inhibitor in their active sites. By contrast, the FEHs that are strongly inhibited by sucrose contain a small glycine or serine residue, probably resulting in a different orientation of their Trp82 homologues (Fig. 2).

It is proposed that the orientation of the bulky Trp 82 might determine the sucrose configuration in the active site of FEHs (Verhaest et al., 2007). Therefore we tried to change the orientation of Trp82 by mutating its neighbouring amino acid serine into a larger apolar amino acid (leucine) in order to prevent sucrose from binding in the inhibitor configuration. Would the change in Trp82 orientation result in a shift from the inhibitor to the substrate configuration? To resolve this tempting issue, the sucrose-hydrolysing activities of the 1-FEH IIa wild-type and S101L mutant enzymes were compared. Fig. 3a clearly demonstrates that a $S 101 L$ mutant is characterized by strongly increased sucrose-hydrolysing activity compared with the wild-type enzyme. However, it should be noted that high amounts of sucrose and enzyme were necessary to observe this invertase activity. At a very low substrate concentration $(5 \mathrm{~mm})$, the introduced sucrosehydrolysing activity should be considered as small compared with the activities towards the preferential 1-FEH IIa substrates 1-kestose and inulin (Fig. 3b). It was demonstrated previously that the $S 101 \mathrm{~L}$ mutant cannot bind sucrose in the inhibitor configuration (Verhaest et al., 2007). Here we demonstrate that sucrose can bind in the substrate configuration, although in a rather inefficient way. Taken together, the data show that sucrose can bind in two distinct configurations: a stable inhibitor configuration (Verhaest et al., 2007) and an unstable substrate configuration (this work). No (or extremely low) catalysis occurs in the stable inhibitor configuration (all sucrose inhibited FEHs), while only very limited catalysis is observed in the unstable substrate configuration. It has been postulated that, when bound in the inhibitor configuration, sucrose cannot be hydrolysed because of the presence of an $\mathrm{H}$-linkage between the acid-base catalyst and the differentially oriented glucosyl moiety of sucrose, hindering the proton donor in its proton donation to the glycosidic oxygen (Verhaest et al., 2007). It is an intriguing question how the unstable substrate configuration (FEHs) can be further converted into a real substrate configuration, as observed in typical plant invertases showing much higher specific activities towards sucrose. For optimal sucrose binding in the substrate configuration and subsequent hydrolysis, it can be assumed that an adapted active site is required, containing some 

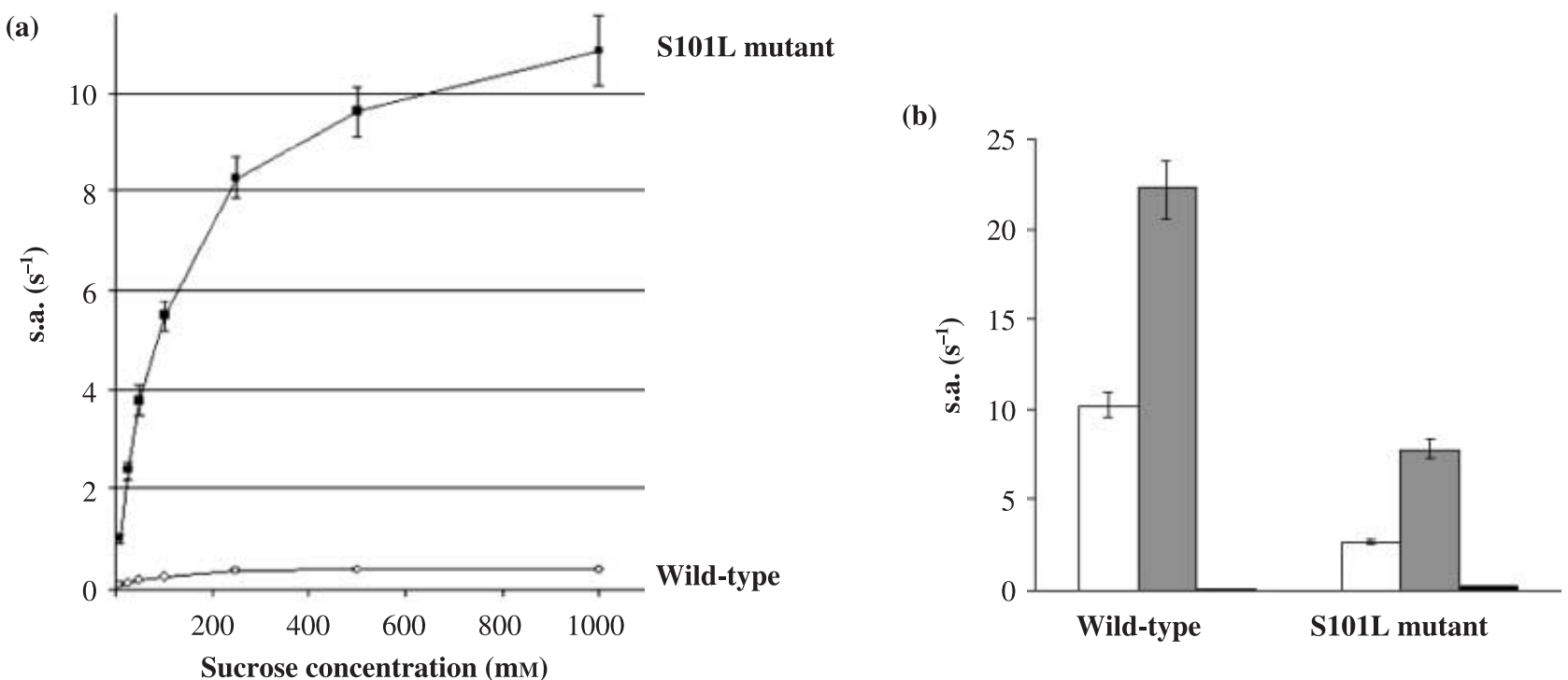

Fig. 3 Specific sucrose-hydrolysing activities (s.a., mol fructose $\mathrm{mol}^{-1}$ enzyme $\mathrm{s}^{-1}$ ) of chicory (Cichorium intybus) fructan 1-exohydrolase (1-FEH Ila) wild-type and S101 L mutant enzymes: (a) in function of increasing substrate concentration (10, 25, 50, 100, 250, 500 and 1000 mM sucrose); (b) when incubated with $5 \mathrm{~mm}$ inulin (I, white bars), 1-kestose (1K, grey bars) and sucrose (S, black bars). Results are means $\pm \mathrm{SE}$ for $n=3$.

additional and/or specific amino acids. Amino acid sequence comparisons, together with examination of the three-dimensional protein structures of both types of enzyme, could help to elucidate why sucrose is efficiently hydrolysed by invertases and not (or in a very limited way) by FEHs.

\section{Introduction of sucrose-hydrolysing activity in $B$. vulgaris 6-FEH}

A closer look at the three-dimensional structures of Arabidopsis cell wall invertase 1 and chicory 1-FEH IIa reveals some marked differences in their active-site regions that might be responsible for the relative (in)capability to bind and hydrolyse sucrose. In AtcwINV1, an additional acidic amino acid Asp239 is observed in the vicinity of the conserved acid-base catalyst Glu203 (Fig. 4a). By contrast, the Asp239 homologue (Glu234) in chicory 1-FEH IIa is twisted over $180^{\circ}$ and oriented away from the active site (Fig. 4b). This different orientation of Glu234 is probably caused by the presence of a double deletion in the vicinity of this residue (Fig. 5). Strikingly, in all known FEHs, such an acidic residue is absent or flanked by a single or double deletion. By contrast, the presence of an Asp239 homologue is highly conserved among cell wall invertases (Fig. 5). It can be questioned whether the introduction of an Asp239 homologue in an FEH would lead to increased invertase activity. As sucrose binds in the inhibitor configuration in chicory 1-FEH IIa and, more importantly, the introduction of a structural and functional Asp239 homologue would be complicated due to the presence of a double deletion in this region, we decided to perform site- directed mutagenesis experiments on B. vulgaris 6-FEH. In this enzyme, sucrose does not bind in the inhibitor configuration (Van den Ende et al, 2003b), and contains no deletions in the vicinity of its Asp239 homologue (Fig. 5). Modelling studies showed that a Phe233Asp substitution in B. vulgaris 6-FEH results in the introduction of a well positioned structural and functional Asp239 homologue (Fig. 4c).

A F233D mutant was constructed and the sucrosehydrolysing activities of both wild-type and F233D mutant 6 -FEH proteins were tested by incubation with increasing amounts of sucrose (up to $1.0 \mathrm{~m}$ final concentration). Fig. 6a shows that the introduction of the aspartate clearly results in a strongly increased sucrose-hydrolysing activity. This result clearly demonstrates for the first time that the introduction of an extra acidic amino acid in the vicinity of the acid-base catalyst can effectively stimulate sucrose binding and catalysis. When compared with the activities against its preferential substrates levan and 6-kestose, an important shift in substrate specificity could be observed for the $F 233 D$ mutant (Fig. 6b). The F233D mutant can be considered as a $\beta$-fructofuranosidase, showing activities against both sucrose and levan. Such enzymes are well known in microbes, but the existence of real $\beta$ fructofuranosidase enzymes in plants is still a matter of debate (Van den Ende et al., 2004). Interestingly, in addition to an introduced sucrose-hydrolysing activity, the F233D mutant enzyme is characterized by a general decrease in activity against levan and 6-kestose, suggesting that Phe233 might play an important role in the overall activity of $B$. vulgaris 6-FEH. It should be noticed that, because of its hydrophobic and aromatic character, this residue could be crucial to stabilize 6-kestose 
New

Phytologist

(a)

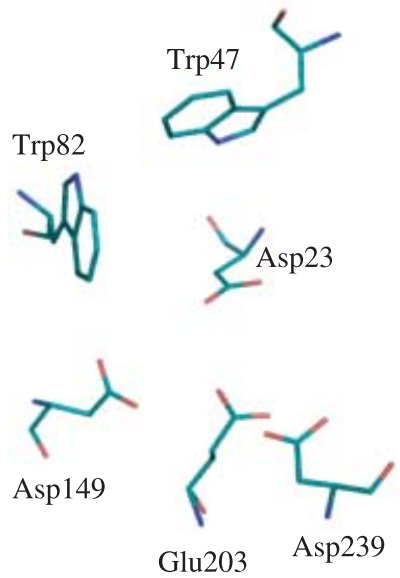

(c) (b)
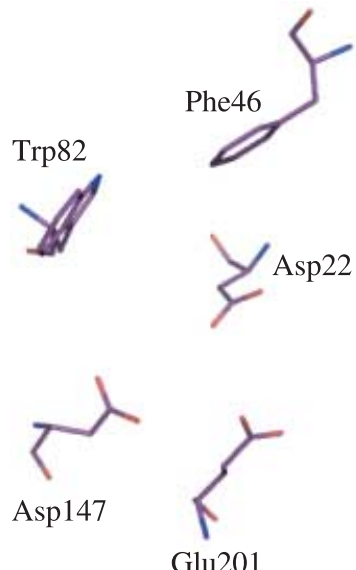

Glu234

Fig. 4 Comparison of the active site of (a) Arabidopsis thaliana cell wall invertase 1 (AtcwINV1) (PDB code 2AC1); (b) chicory (Cichorium intybus) fructan 1-exohydrolase (1-FEH Ila) (PDB code 1ST8); (c) Beta vulgaris fructan 6-exohydrolase (6-FEH), showing the presence/absence of an Asp239 and Trp47 structural and functional homologue. Modelling of $B$. vulgaris 6-FEH was performed based on the known three-dimensional structure of AtcwINV1 using sWISS MODEL (http:// swissmodel.expasy.org). Figures were prepared with PYMOL (Delano, 2002).

and higher DP levan substrate in the active site. It is well known that aromatic residues can play an important role by stabilizing the sugar-enzyme complex (Nagy et al., 1998; Van der Veen et al., 2001).

Although the introduction of an additional acidic residue in the active site of $B$. vulgaris 6-FEH caused a significant increase in sucrose-hydrolysing activity in the F233D mutant, the preference for levan and 6-kestose as a substrate remained. This suggests that still some other amino acids might play a crucial role in the optimal binding and hydrolysis of sucrose. Recent structure-function work on AtcwINV1 shows an important role of the Trp47 residue in stabilizing sucrose in the active site (Le Roy et al., 2007b). The presence of a Trp47 homologue is highly conserved among cell wall invertases, in contrast to plant FEHs (Fig. 5). Interestingly, a Trp residue is also present in $B$. vulgaris 6-FEH, but modelling studies show that this Trp45 might be oriented away from the active site due to a tight stacking interaction with His46 (Fig. 4c). This could reasonably explain the observed suboptimal binding of sucrose in the active site of the $F 233 D$ mutant. It should also be noticed that 1-FEH IIa contains a well oriented Phe46 homologue at this position, which could assist in stabilizing sucrose in the S101L 1-FEH IIa mutant, even in the absence of an Asp239 homologue (Fig. 4b).

\section{Functional diversification and evolution of enzyme specificity}

Taken together, the data presented by Le Roy et al. (2007b) and in this manuscript greatly support the idea that only a few amino acids determine the difference in substrate specificity between FEHs and invertases. It can be speculated that plant FEHs and invertases, each with their characteristic substrate specificities, were derived from an ancestral $\beta$-fructofuranosidase type of enzyme, as still present in microorganisms today. One of our mutant enzymes $(F 233 D)$ behaved as a typical $\beta$-fructofuranosidase, but it is unclear whether such an 'evolutionary intermediate' exists in plants today. Whatever the exact process of enzyme speciation throughout evolution, apparently the uncoupling of sucrose and fructan metabolism 


\begin{tabular}{|c|c|c|}
\hline CWINV1 A. thaliana & WGN & WECPD \\
\hline CWINV2 A. thaliana & WGN & WECPD \\
\hline CWINV4 A. thaliana & WGN & WECPD \\
\hline CWINV1 $s$. tuberosum & WGN & WECPD \\
\hline CWINV2 $S$. tuberosum & WGN & WECPD \\
\hline CWINV3 S. tuberosum & WGN & WECPD \\
\hline CWINVLin5 L. esculentum & WGN & WECPD \\
\hline CWINV $P$. sativum & WGN & WECPD \\
\hline CWINV1 V. faba & WGN & WECPD \\
\hline CWINV2 V. faba & WGN & WECPD \\
\hline CWINV $V$. vinifera & WGN & WECPD \\
\hline CWINV1 D. carota & WGN & WECPD \\
\hline CWINV C. papaya & WGN & WECPD \\
\hline CWINV $M$. acuminata & WGN & WECPD \\
\hline CWINV1 Z. mays & WGN & WECPD \\
\hline CWINV2 Z. mays & WGN & WECPD \\
\hline CWINV3 Z. mays & WGN & WECPD \\
\hline CWINV L. perenne & WGN & WECPD \\
\hline CWINV T. aestivum & WGN & WECPD \\
\hline 1-FEH IIa/b C. intybus & FGD & WECPD \\
\hline 1-FEH I C. intybus & WGN & WECPD \\
\hline 1-FEH C. rapunculoides & FGD & WECPD \\
\hline 1-FEH w1/w2 T.aestivum & FGD & WECPD \\
\hline 1-FEH L. perenne & WGN & WECPD \\
\hline $6 \& 1-F E H$ A. thaliana & FSR & WECPD \\
\hline 6\&1-FEH T. aestivum & $\mathrm{DPN}$ & WECLD \\
\hline 6-FEH A. thaliana & WDV & WECPD \\
\hline 6-FEH B. vulgaris & WHT & WECPD \\
\hline 6-FEH T. aestivum & WQP & VECPD \\
\hline 6-KEH w1 T. aestivum & NPN & FECLD \\
\hline 6-FEH w2 T. aestivum & NPN & LECLD \\
\hline
\end{tabular}

239

KISLDDTKH

KVSLDLTRY

KVSLDITRY

KVSFDVTRF

KNSMDLTRF

KNSLDVNRF

KNSLDVARF

KVSLDDTKH

KNSLDITRY

KVSLDDKKH

KLSLDDTKH

KVSLDLTRY

KVSLDLTRY

KVSLDLRKY

KSSLDLTRY

KNSLDLRRY

KVSLDVTRY

KNSLDLTRY

KVSLDVTRY

KAGFEG- -H KVSFNS- - R KVGFNG- - I

KMSVDS - - V

KMSLDS - -S

KASFGG - - N

KVSINS- - C

KVSLIETLH

KLSLFDTQY

KLSVMNTTQ

KMGMNF-GE

KMGLNF-GE
Fig. 5 Multiple sequence alignment of the Trp47, Glu203 (acid-base catalyst) and Asp239 region in plant cell wall invertases and fructan exohydrolases. The following cDNA-deduced amino acid sequences (with according accession number) are used for alignment: Arabidopsis thaliana cell wall invertase 1 (AY079422), cell wall invertase 2 (AK118343), invertase 4 (AB049617), 6\&1-FEH (AY060533) and 6-FEH (AB029310); Solanum tuberosum cell wall invertase 1 (Q43171), cell wall invertase 2 (Q9M4K7) and cell wall invertase 3

(Q9M4K8); Lycopersicon esculentum cell wall invertase Lin5 (AY17350); Pisum sativum cell wall invertase (AF063246); Vicia faba cell wall invertase 1 (Z35162) and cell wall invertase 2 (Z35163); Vitis vinifera cell wall invertase (AY538262); Daucus carota cell wall invertase 1 (×69 321); Carica papaya cell wall invertase (AF420223); Musa acuminata cell wall invertase (AY180200); Zea mays cell wall invertase 1 (AF050129), cell wall invertase 2 (AF050128) and cell wall invertase 3(AF050631); Triticum aestivum cell wall invertase (AF030421); Lolium perenne cell wall invertase (DQ073969); Cichorium intybus 1-FEH Ila (AJ295033), 1-FEH IIb (AJ295034) and 1-FEH I (AJ242538); Campanula rapunculoides 1-FEH (AJ509808); Triticum aestivum 1-FEH w1 (AJ 516025), 1-FEH w2 (AJ508387), 6\&1-FEH (AB089269), 6-FEH (AM075205), 6-KEH w1 (AB089271) and 6-KEH w2 (AB089270); Lolium perenne 1-FEH (DQ016297).
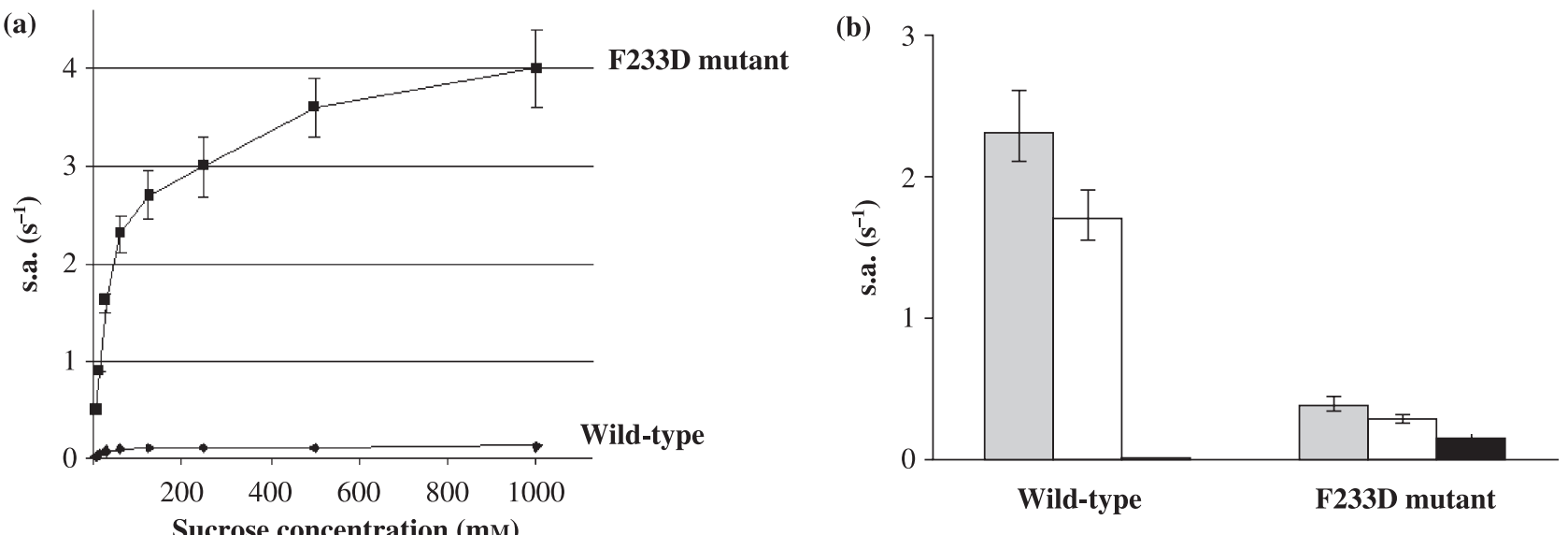

Fig. 6 Specific sucrose-hydrolysing activities (s.a., mol fructose $\mathrm{mol}^{-1}$ enzyme $\mathrm{s}^{-1}$ ) of Beta vulgaris fructan 6-exohydrolase (6-FEH) wild-type and F233D mutant enzymes: (a) as a function of increasing substrate concentration (10, 25, 50, 100, 250, 500 and $1000 \mathrm{~mm}$ sucrose); (b) when incubated with $5 \mathrm{~mm}$ levan (L, grey bars), 6-kestose (6K, white bars) and sucrose (S, black bars). Results are means \pm SE for $n=3$. 
turned out to be highly beneficial in plants, and in this respect the most important characteristic of FEHs in nonfructan plants might be their inability to degrade sucrose, putatively allowing them to fulfil a regulatory function in a high-sucrose background. It should be noted that the mutations leading to enzyme speciation might be situated not only in the active site region (e.g. removal of Asp239 homologue to create a FEH), but probably also in the cavity between the two structural domains in family 32 members. It was recently demonstrated by site-directed mutagenesis on FEHs that this cleft plays an important role in stabilizing higher DP fructans as a donor substrate in this cleft (Le Roy et al., 2007a).

\section{Conclusion}

For the first time, invertase activity was successfully introduced in two different FEH enzymes by specific site-directed mutagenesis. Our results strongly suggest that a correct orientation of $\operatorname{Trp} 82$ (avoiding binding of sucrose in the inhibitor configuration), and the presence of an Asp 239 homologue, are two essential requirements for stabilizing sucrose in the active site, although some additional amino acids (e.g. Trp45) are probably necessary to achieve optimal binding and catalysis of sucrose. Especially the absence or presence of an Asp239 homologue appears to be particularly important in understanding the functional difference between FEHs and invertases. This point of view is consistent with the fact that all functionally characterized FEHs lack an Asp239 homologue and are unable to degrade sucrose. On the other hand, an Asp239 homologue is found in all plant cell wall invertases, although few of them are functionally characterized. More research is needed to further test this hypothesis on a wider array of GH32 members, including microbial enzymes. As only a few mutations can affect donor substrate specificity, our results suggest that plant invertases in FEHs may have diversified by introduction of a very limited number of mutations in the common ancestor.

\section{Acknowledgements}

Wim Van den Ende is supported by a grant from the Fund for Scientific Research (FSR Flanders). The authors are very grateful to Rudy Vergauwen for his technical assistance.

\section{References}

Asega AF, Carvalho MAM. 2004. Fructan metabolizing enzymes in rhizophores of Vernonia herbacea upon excision of aerial organs. Plant Physiology and Biochemistry 42: 313-319.

De Coninck B, Le Roy K, Francis I, Clerens S, Vergauwen R, Halliday AM, Smith SM, Van Laere A, Van den Ende W. 2005. Arabidopsis AtcwINV3 and 6 are not invertases but are fructan exohydrolases (FEHs) with different substrate specificities. Plant, Cell \& Environment 28: 432-443.

De Roover J, De Winter M, Van Laere A, Timmermans JW, Van den Ende W. 1999. Purification and properties of a second fructan exohydrolase from the roots of Cichorium intybus L. Physiologia Plantarum 106: 28-34.

Delano WL. 2002. The PyMOL molecular graphics system. San Carlos, CA, USA: Delano Scientific, http://www.pymol.org.

Hendry GAF, Wallace RK. 1993. The origin, distribution and evolutionary significance of fructans. In: Suzuki M, Chatterton NJ, eds. Science and technology of fructans. Boca Raton, FL, USA: CRC Press, 119-139.

Henrissat B, Davies G. 1997. Structural and sequence-based classification of glycoside hydrolases. Current Opinion in Structural Biology 7: 637-644.

Iizuka M, Yamaguchi H, Ono S, Minimiura N. 1993. Production and isolation of levan by use of levansucrase immobilized on the ceramic support SM-10. Bioscience, Biotechnology \& Biochemistry 57: 322-324.

Kawakami A, Yoshida M, Van den Ende W. 2005. Molecular cloning and functional analysis of a novel 6\&1-FEH from wheat (Triticum aestivum $\mathrm{L}$.) preferentially degrading small branched graminans like bifurcose. Gene 358: 93-101.

Koch KE. 1996. Carbohydrate-modulated gene expression in plants. Annual Review of Plant Physiology and Plant Molecular Biology 47: 509-540.

Lasseur B, Lothier J, Djoumad A, De Coninck B, Smeekens S, Van Laere A, Morvan-Bertrand A, Van den Ende W, Prud'homme M-P. 2006. Molecular and functional characterization of a cDNA encoding fructan:fructan 6G-fructosyltransferase (6G-FFT)/fructan:fructan 1 -fructosyltransferase (1-FFT) from perennial ryegrass (Lolium perenne L.). Journal of Experimental Botany 57: 2719-2734.

Le Roy K, Lammens W, Verhaest M, De Coninck B, Rabijns A, Van Laere A, Van den Ende W. 2007b. Unraveling the difference between invertases and fructan exohydrolases: a single amino acid (Asp-239) substitution transforms Arabidopsis cell wall invertase1 into a fructan 1-exohydrolase. Plant Physiology 145: 616-625.

Le Roy K, Verhaest M, Rabijns A, Clerens S, Van Laere A, Van den Ende W. 2007a. $N$-glycosylation affects substrate specificity in chicory 1-FEH IIa: evidence for the presence of an inulin binding cleft. New Phytologist 176: 317-324.

Lewis DH 1993. Nomenclature and diagrammatic representation of oligomeric fructans - a paper for discussion. New Phytologist 124: 583594.

Lothier J, Lasseur B, Le Roy K, Van Laere A, Prud'homme M-P, Barre P, Van den Ende W, Morvan-Bertrand A. 2007. Cloning, gene mapping, and functional analysis of a fructan 1-exohydrolase (1-FEH) from Lolium perenne implicated in fructan synthesis rather than in fructan mobilization. Journal of Experimental Botany 58: 1969-1983.

Meng G, Fütterer K. 2003. Structural framework of fructosyltransfer in Bacillus subtilis levansucrase. Nature Structural Biology 10: 935-941.

Morvan-Bertrand A, Boucaud J, Le Soas J, Prud'homme MP. 2001. Roles of the fructans from leaf sheats and from the elongating leaf bases in the regrowth following defoliation of Lolium perenne L. Planta 213: $109-120$.

Nagy T, Simpson P, Williamson MP, Hazlewood GP, Gilbert HJ, Orosz L. 1998. All three surface tryptophans in type IIa cellulose binding domains play a pivotal role in binding of both soluble and insoluble ligands. FEBS Letters 429: 312-316.

Naumoff DG. 2001. $\beta$-fructosidase superfamily: homology with some $\alpha$-L-arabinases and $\beta$-D-xylanases. Proteins 42: 66-76.

Reddy A, Maley F. 1996. Studies on identifying the catalytic role of Glu-204 in the active site of yeast invertase. Journal of Biological Chemistry 271: 13953-13957.

Reddy VA, Maley F. 1990. Identification of an active-site residue in yeast invertase by affinity labeling and site-directed mutagenesis. Journal of Biological Chemistry 265: 10817-10820.

Sedmak JJ, Grossberg SE. 1977. Rapid, sensitive and versatile assay for protein using Coomassie Brilliant Blue G250. Analytical Biochemistry 79: $544-552$.

Shiomi N. 1989. Properties of fructosyltransferases involved in the synthesis of fructan in Liliaceous plants. Journal of Plant Physiology 134: 151-155. 
Van den Ende W, Clerens S, Vergauwen R, Van Riet L, Van Laere A, Yoshida M, Kawakami A. 2003a. Fructan 1-exohydrolases. $\beta(2,1)$ trimmers during graminan biosynthesis stems of wheat? Purification, characterization, mass mapping and cloning of two fructan 1-exohydrolase isoforms. Plant Physiology 131: 621-631.

Van den Ende W, De Coninck B, Clerens S, Vergauwen R, Van Laere A. 2003b. Unexpected presence of fructan exohydrolases (6-FEHs) in non-fructan plants: characterization, cloning, mass mapping and functional analysis of a novel 'cell-wall invertase-like' specific 6-FEH from sugar beet (Beta vulgaris L.). Plant Journal 36: 697-710.

Van den Ende W, De Coninck B, Van Laere A. 2004. Plant fructan exohydrolases: a role in signaling and defense? TRENDS in Plant Science 9: 523-528.

Van den Ende W, Michiels A, De Roover J, Verhaert P, Van Laere A. 2000. Cloning and functional analysis of chicory root fructan 1-exohydrolase I (1-FEH I): a vacuolar enzyme derived from a cell-wall invertase ancestor? Mass fingerprint of the 1-FEH I enzyme. Plant Journal 24: 447-456.

Van den Ende W, Michiels A, Van Wonterghem D, Clerens SP, De Roover J, Van Laere A. 2001. Defoliation induces fructan 1 -exohydrolase II in witloof chicory roots. Cloning and purification of two isoforms, fructan 1-exohydrolase IIa and fructan 1-exohydrolase IIb. Mass fingerprint of the fructan exohydrolase II enzymes. Plant Physiology 126: 1186-1195.

Van den Ende W, Van Laere A. 1996. Fructan synthesizing and degrading activities in chicory roots (Cichorium intybus L.) during growth, storage and forcing. Journal of Plant Physiology 149: 43-50.

Van der Veen BA, Leemhuis H, Kralj S, Uitdehaag JC, Dijkstra BW, Dijkhuizen L. 2001. Hydrophobic amino acid residues in the acceptor binding site are main determinants for reaction mechanism and specificity of cyclodextrin-glycosyltransferase. Journal of Biological Chemistry 276: 44557-44562.

Van Laere A, Van den Ende W. 2002. Inulin metabolism in dicots: chicory as a model system. Plant, Cell \& Environment 25: 803-813.

Van Riet L, Nagaraj V, Van den Ende W, Clerens S, Wiemken A, Van Laere A. 2006. Purification, cloning and functional characterization of a fructan 6-exohydrolase from wheat (Triticum aestivum L.). Journal of Experimental Botany 57: 213-223.

Vandamme EJ, Derycke DG. 1983. Microbial inulinases: fermentation process, properties, and applications. Advances in Applied Microbiology 29: 139-176.

Verhaest M, Lammens W, Le Roy K, De Ranter CJ, Van Laere A, Rabijns A, Van den Ende W. 2007. Insights into the fine architecture of the active site of chicory fructan 1-exohydrolase: 1-kestose as substrate vs sucrose as inhibitor. New Phytologist 174: 90-100.

Verhaest M, Lammens W, Le Roy K, De Ranter CJ, Van Laere A, Van den Ende W, Rabijns A. 2006. X-ray diffraction structure of a cell wall invertase from Arabidopsis thaliana. Acta Crystallographica Section D 62: $1555-1563$

Verhaest M, Van den Ende W, Le Roy K, De Ranter CJ, Van Laere AV, Rabijns A. 2005. X-ray diffraction structure of a plant glycosyl hydrolase family 32 protein: fructan 1-exohydrolase IIa of Cichorium intybus. Plant Journal 41: 400-411.

Verhaest M, Van den Ende W, Yoshida M, Le Roy K, Peeraer Y, Sansen S, De Ranter CJ, Van Laere A, Rabijns A. 2004. Crystallization and preliminary $\mathrm{X}$-ray diffraction study of fructan 1-exohydrolase from Cichorium intybus. Acta Crystallographica Section D 60: 553-554.

Vijn I, Smeekens S. 1999. Fructan: more than a reserve carbohydrate? Plant Physiology 120: 351-359.

\section{Pbout New Phytologist}

- New Phytologist is owned by a non-profit-making charitable trust dedicated to the promotion of plant science, facilitating projects from symposia to open access for our Tansley reviews. Complete information is available at www.newphytologist.org.

- Regular papers, Letters, Research reviews, Rapid reports and both Modelling/Theory and Methods papers are encouraged. We are committed to rapid processing, from online submission through to publication 'as-ready' via OnlineEarly - our average submission to decision time is just 28 days. Online-only colour is free, and essential print colour costs will be met if necessary. We also provide 25 offprints as well as a PDF for each article.

- For online summaries and ToC alerts, go to the website and click on 'Journal online'. You can take out a personal subscription to the journal for a fraction of the institutional price. Rates start at $£ 135$ in Europe/\$251 in the USA \& Canada for the online edition (click on 'Subscribe' at the website).

- If you have any questions, do get in touch with Central Office (newphytol@lancaster.ac.uk; tel +44 1524 594691) or, for a local contact in North America, the US Office (newphytol@ornl.gov; tel +1 8655765261 ). 